\title{
Mice with Altered Myelin Proteolipid Protein Gene Expression Display Cognitive Deficits Accompanied by Abnormal Neuron-Glia Interactions and Decreased Conduction Velocities
}

\author{
Hisataka Tanaka, ${ }^{1 \star}$ Jianmei Ma, ${ }^{1,5 *}$ Kenji F. Tanaka, ${ }^{1 \star}$ Keizo Takao, ${ }^{6,7}$ Munekazu Komada, ${ }^{2,10}$ Koichi Tanda, ${ }^{6}$ \\ Ayaka Suzuki, ${ }^{8}$ Tomoko Ishibashi, ${ }^{8}$ Hiroko Baba, ${ }^{8}$ Tadashi Isa, ${ }^{3}$ Ryuichi Shigemoto, ${ }^{4}$ Katsuhiko Ono, ${ }^{9}$ \\ Tsuyoshi Miyakawa, ${ }^{2,6,7,10}$ and Kazuhiro Ikenaka ${ }^{1,10}$ \\ ${ }^{1}$ Division of Neurobiology and Bioinformatics, ${ }^{2}$ Center for Genetic Analysis of Behavior, ${ }^{3}$ Department of Developmental Physiology, and ${ }^{4}$ Division of \\ Cerebral Structure, National Institute for Physiological Sciences, National Institutes of Natural Sciences, Okazaki, Aichi 444-8787, Japan, ${ }^{5}$ Department of \\ Anatomy, Dalian Medical University, Dalian, Liaoning 116044, China, ${ }^{6}$ Genetic Engineering and Functional Genomics Group, Horizontal Medical Research \\ Organization, Kyoto University Faculty of Medicine, Sakyo-ku, Kyoto 606-8501, Japan, ${ }^{7}$ Division of Systems Medicine, Institute for Comprehensive Medical \\ Science, Fujita Health University, Aichi 470-1192, Japan, ${ }^{8}$ Department of Molecular Neurobiology, School of Pharmacy, Tokyo University of Pharmacy and \\ Life Sciences, Hachioji 192-0392, Japan, ${ }^{9}$ Department of Biology, Kyoto Prefectural University of Medicine, Kyoto 602-8566, Japan, and ${ }^{10}$ Japan Science and \\ Technology Agency, Core Research for Evolutionary Science and Technology, Kawaguchi 332-0012, Japan
}

Conduction velocity (CV) of myelinated axons has been shown to be regulated by oligodendrocytes even after myelination has been completed. However, how myelinating oligodendrocytes regulate $\mathrm{CV}$, and what the significance of this regulation is for normal brain function remain unknown. To address these questions, we analyzed a transgenic mouse line harboring extra copies of the myelin proteolipid protein 1 ( $p l p 1)$ gene ( $p l p 1^{\mathrm{tg} /-}$ mice) at 2 months of age. At this stage, the $p l p 1^{\mathrm{tg} /-}$ mice have an unaffected myelin structure with a normally appearing ion channel distribution, but the $\mathrm{CV}$ in all axonal tracts tested in the CNS is greatly reduced. We also found decreased axonal diameters and slightly abnormal paranodal structures, both of which can be a cause for the reduced CV. Interestingly the $p l p 1^{\mathrm{tg} /-}$ mice showed altered anxiety-like behaviors, reduced prepulse inhibitions, spatial learning deficits and working memory deficit, all of which are schizophrenia-related behaviors. Our results implicate that abnormalities in the neuron-glia interactions at the paranodal junctions can result in reduced CV in the CNS, which then induces behavioral abnormalities related to schizophrenia.

\section{Introduction}

Myelin is a lipid-rich multilayered structure that wraps and insulates axons, providing them with an increased conduction velocity (CV) (Waxman and Bangalore 2005). Recent studies have demonstrated that myelination of an axon induces a dynamic change in axonal membrane protein localization, and segregates the axonal membrane into segments called the node of Ranvier, paranode, juxtaparanode, and internode (Poliak and Peles, 2003). The CV and localization of axonal membrane proteins are known to be altered under certain pathological conditions, including demyelinating diseases, but they have not been shown to

Received July 9, 2008; revised May 8, 2009; accepted May 25, 2009.

This work was supported by Grant-in Aid \#15082209 for Scientific Research on Priority Areas on "Elucidation of glia-neuron network mediated information processing systems" and on "IBR-SHIEN" from Ministry of Education, Culture, Sports, Science and Technology to K.I., T.M., R.S., and H.B. We thank Professor E. Peles for kindly providing us with anti-Caspr antibody and for grateful advice; and G. Yamada, R. Taguchi, and S. Yamada for technical assistance.

*H.T., J.M., and K.F.T. contributed equally to this work.

Correspondence should be addressed to Kazuhiro Ikenaka, National Institute for Physiological Sciences, 5-1 Higashiyama, Myodaiji, 0kazaki 444-8787, Japan. E-mail: ikenaka@nips.ac.jp.

DOI:10.1523/JNEUROSCI.3216-08.2009

Copyright $\odot 2009$ Society for Neuroscience $\quad$ 0270-6474/09/298363-09\$15.00/0 vary under nondemyelinating conditions. Recently Yamazaki et al. (2007) showed that activation of oligodendrocytes could alter the $\mathrm{CV}$ of the axons it myelinates. Thus, the relationship between myelin and axon may not be stationary, but may in fact be dynamic. However, the cause for this altered CV, or the behavioral consequence induced by altering the $\mathrm{CV}$ have not yet been studied.

We have recently shown that at 2 months of age, CV in a plp 1 transgenic mouse line [ $p l p 1$ transgenic mouse line 4 e hemizygote; $p l p 1^{t g /-}$ mice (Kagawa et al., 1994)] is reduced by half, and relative refractory periods (RRPs) are prolonged in all 3 axonal tracts tested (dorsal column pathway, vestibulospinal/reticulospinal tracts (VRST), and pyramidal tract) in the CNS (CNS) (Tanaka et al., 2006). Previous studies demonstrated that at 2 months of age, the myelin in these mice was intact (Inoue et al., 1996), and $\mathrm{Na}^{+}$ and $\mathrm{K}^{+}$channel clusters were formed normally at the node of Ranvier and its adjacent region, respectively (Ishibashi et al., 2003; Rasband et al., 2003). Although, at a much later stage, these mice undergo demyelination (Kagawa et al., 1994). We were curious to know what the cause of this decreased $\mathrm{CV}$ is, and how the abnormal conduction in the CNS would affect the behavior of 
these mice. In this study, we performed extensive analyses on the behavior of 2-month-old $p l p 1^{t g /-}$ mice, and found that they displayed various behavioral abnormalities indicative of cognitive dysfunction that may be interpreted as schizophrenia-like behavior. We also found abnormal neuron-glia interactions, which could provoke the abnormal conduction. This result provides a possible mechanism that describes the pathophysiology of schizophrenia.

\section{Materials and Methods}

Animals

The plp 1 transgenic mouse line $4 \mathrm{e}$ was generated by introducing a cosmid clone containing the entire mouse plpl gene (Kagawa et al., 1994). For our studies, we used wild-type BDF1 mice (Wt) and the hemizygous plp1 transgenic mice harboring two extra copies of the wild-type plp1 gene $\left(p l p 1^{t g /-}\right)$ in the BDF1 background. These strains were maintained and propagated by mating with BDF1 mice (Charles River Japan) in the Center for Experimental Animals, National Institutes of Natural Sciences. The animal research protocol was approved by the Institutional Animal Care and Use committee.

\section{Behavioral analysis}

Experimental design. Mice were divided into three groups. The first group of mice was used in a comprehensive battery of behavioral tests (Crawley, 2000; Yamasaki et al., 2008). The battery included the neurological screen, wire hang, grip strength, open field, light/dark transition, hot plate, social interaction, prepulse inhibition, Porsolt forced swim, and elevated plus maze tests. These tests were conducted in the sequence listed, with each test separated at least by $1 \mathrm{~d}$ ( $p l p 1^{\text {tg/- }}$ mice, $n=21 ; \mathrm{Wt}$ littermates, $n=21)$. The second group of mice was used for the Barnes spatial navigation task ( $p l p 1^{\text {tg/- }}$ mice, $n=9$; Wt littermates, $n=9$ ) (Takao et al., 2008). The third group of mice was used for the T-maze forced alternation task ( $p l p 1^{\text {tg/- }}$ mice, $n=23$; Wt littermates, $n=22$ ) (Takao et al., 2008). The behavioral tests of the second and third groups were performed with male mice that were 10 weeks old at the start of the testing. Mice were housed three to five per cage in a room with a $12 \mathrm{~h}$ light/dark cycle (lights on at 6:00 A.M.) with ad libitum access to food and water. Behavioral testing was performed between 8:00 A.M. and 6:00 P.M. The raw data of behavioral tests, which are not described in this study, are disclosed in the Mouse Phenotype Database https://behav.hmro.med.kyoto-u.ac.jp/.

Wire hang test. A wire hang test apparatus (Ohara) was used to assess balance and grip strength. The apparatus consists of a box $(21.5 \times 22 \times$ $23 \mathrm{~cm})$ and a wire mesh grid $(10 \times 10 \mathrm{~cm})$ on its top, which can be inverted. The mouse was placed on a wire mesh, which was then inverted, so that the subject gripped the wire. Latency to fall (s) was recorded, with a 60 s cutoff time.

Grip strength test. A grip strength meter (Ohara) was used to assess forelimb grip strength. Mice were lifted and held by their tail so that their forepaws could grasp a wire grid. The mice were then gently pulled backward by the tail with their posture parallel to the surface of the table until they released the grid. The peak force applied by the forelimbs of the mouse was recorded in Newtons $(\mathrm{N})$. Each mouse performed the test three times, and the greatest value measured was used for statistical analysis.

Open field test. Locomotor activity was measured using an open field test. Each subject was placed in the center of the open field apparatus $(40 \times 40 \times 30 \mathrm{~cm}$; Accuscan Instruments). Total distance traveled (in $\mathrm{cm}$ ), vertical activity (rearing measured by counting the number of photobeam interruptions), and time spent in the center (s) were recorded. Data were collected for $30 \mathrm{~min}$.

Light/dark transition test. The apparatus used for the light/dark transition test consisted of a cage $(21 \times 42 \times 25 \mathrm{~cm})$ divided into two sections of equal size by a partition with a door (Ohara). One chamber was brightly illuminated (390 lux), whereas the other was dark (2 lux). Mice were placed into the dark side and allowed to move freely between the two chambers with door open for $10 \mathrm{~min}$. The total number of transitions and time spent in each side were recorded by Image LD software (see Image analysis).
Hot plate test. The hot plate test was used to evaluate the sensitivity to a painful stimulus. Mice were placed on a $55.0^{\circ} \mathrm{C}\left(0.3^{\circ} \mathrm{C}\right)$ hot plate $(\mathrm{Co}-$ lumbus Instruments), and latency to the first paw response (s) was recorded. The paw response was either a foot shake, or a paw lick, or lifting both forepaws simultaneously.

Social interaction test in a novel environment. In the social interaction test, two mice of identical genotypes, which were previously housed in different cages, were placed together in a box $(40 \times 40 \times 30 \mathrm{~cm})$, and allowed to explore freely for $10 \mathrm{~min}$. Social behavior was monitored by a CCD camera, which was connected to a Macintosh computer. Analysis was performed automatically using Image SI software (see Image analysis). The number of total contacts, mean duration per contact (s), and total distance traveled $(\mathrm{cm})$ were measured.

Startle response/prepulse inhibition tests. A startle reflex measurement system was used (Ohara). A test session began by placing a mouse in a Plexiglas cylinder where it was left undisturbed for $10 \mathrm{~min}$. We used white noise as the startle stimulus that lasted $40 \mathrm{~ms}$ for all trial types. The startle response was recorded for $140 \mathrm{~ms}$ (measuring the response every 1 $\mathrm{ms}$ ) starting with the onset of the prepulse stimulus. The background noise level in each chamber was $70 \mathrm{~dB}$. The peak startle amplitude recorded during the $140 \mathrm{~ms}$ sampling window was used as the dependent variable. A test session consisted of 6 trial types (i.e., two types for startle stimulus only trials, and four types for prepulse inhibition trials). The intensity of the startle stimulus was 110 or $120 \mathrm{~dB}$. The prepulse sound was presented $100 \mathrm{~ms}$ before the startle stimulus, and its intensity was 74 or $78 \mathrm{~dB}$. Four combinations of prepulse and startle stimuli were used $(74-110,78-110,74-120$, and $78-120)$. Six blocks of the 6 trial types were presented in pseudorandom order such that each trial type was presented once within a block. The average intertrial interval was $15 \mathrm{~s}$ (range: 10-20 s).

Porsolt forced swim test. In the Porsolt forced swim test, the apparatus consisted of four Plexiglas cylinders $(20 \mathrm{~cm}$ height $\times 10 \mathrm{~cm}$ diameter $)$. The cylinders were separated from each other by a nontransparent panel to prevent mice from seeing each other. The cylinders were filled with water $\left(23^{\circ} \mathrm{C}\right)$, up to a height of $7.5 \mathrm{~cm}$. Mice were placed into the cylinders, and their behavior was recorded over a 10 -min test period. Data acquisition and analysis were performed automatically, using Image PS software (Image analysis).

Elevated plus-maze test. The elevated plus-maze consisted of two open arms $(25 \times 5 \mathrm{~cm})$ and two enclosed arms of the same size, with $15-\mathrm{cm}-$ high transparent walls. The arms and central square were made of white plastic plates, and were elevated to a height of $55 \mathrm{~cm}$ above the floor. To minimize the likelihood of animals falling from the apparatus, 3-mmhigh Plexiglas ledges were provided for the open arms. Arms of the same type were arranged at opposite sides to each other. Each mouse was placed in the central square of the maze $(5 \times 5 \mathrm{~cm})$, facing one of the closed arms. Mouse behavior was recorded during a $10 \mathrm{~min}$ test period. The number of entries into, and the time spent on open and enclosed arms were recorded. For data analysis, we used the following four measures: the percentage of entries into open arms, the stay time on open $\operatorname{arms}(\mathrm{s})$, the number of total entries, and total distance traveled $(\mathrm{cm})$. Data acquisition and analysis were performed automatically, using Image EP (see Image analysis).

The Barnes spatial navigation task. The Barnes task was conducted on "dry land," a white circular surface, $1.0 \mathrm{~m}$ in diameter, with 12 holes equally spaced around the perimeter (Ohara). The circular open field was elevated $75 \mathrm{~cm}$ from the floor. A black Plexiglas escape box $(17 \times 13 \times 7$ $\mathrm{cm}$ ), which had paper cage bedding on its bottom, was located under one of the holes. The hole above the escape box represented the target, analogous to the hidden platform in the Morris task. The location of the target was consistent for a given mouse but randomized across mice. The maze was rotated daily, with the spatial location of the target unchanged with respect to the distal visual room cues, to prevent a bias based on olfactory or the proximal cues within the maze. Four trials per day were conducted for 5 successive days. On day 6, a probe trial was conducted without the escape box, to confirm that this spatial task was acquired based on navigation by distal environment room cues. Latency to reach the target hole, distance to reach the target hole, number of errors, and 
time spent around each hole were recorded by videotracking Image BM software (see Image analysis).

T-maze forced alternation task. The forced alternation task was conducted using an automatic T-maze (Ohara). The maze was partitioned into the right and left arms $(11.5 \times 20.5 \mathrm{~cm})$ and the stem $(13 \times 24 \mathrm{~cm})$ area by automated sliding doors. The end of each arm was equipped with a pellet dispenser that could provide food reward. One week before the pretraining, mice were deprived of food until their body weight was reduced to $80-85 \%$ of the initial level. Before the first trial, mice were subjected to three 10-min adaptation sessions. On the day after the adaptation session, mice were subjected to a forced alternation protocol for $16 \mathrm{~d}$ (one session consisting of 10 trials per day; cutoff time, $50 \mathrm{~min}$ ). On the first (sample) trial, the mouse was forced to choose one of the arms, and received the reward at the end of the arm. Choosing the incorrect arm resulted in no reward and confinement to the arm for $10 \mathrm{~s}$. After the mouse consumed the pellet or the mouse stayed $>10$ s without consuming the pellet, the mouse returned to the starting gate of stem area. The mouse was then given $3 \mathrm{~s}$ delay there and a free choice between both $\mathrm{T}$ arms and rewarded for choosing the other arm that was not chosen on the first trial of the pair. The location of the sample arm (left or right) was varied pseudo-randomly across trials. A variety of fixed extra-maze clues surrounded the apparatus. On days $16-21$, delay (10, 30 or $60 \mathrm{~s})$ was applied after the sample trial. Data acquisition, control of sliding doors, and data analysis were performed by Image TM software (see Image analysis).

Image analysis. All applications used for the behavioral studies (Image EP, Image BM, Image SI, Image LD, Image OF, Image PS, and Image TM) were run on a Macintosh computer. Applications were based on the public domain NIH Image program (developed by Wayne Rasband at the U.S. National Institute of Mental Health and available on the Internet at http://rsb.info.nih.gov/nih-image/) and were modified for each test by Tsuyoshi Miyakawa (available through Ohara).

Statistical analysis. Statistical analysis was conducted using StatView (SAS Institute). Data were analyzed by two-way ANOVA, or two-way repeated measures ANOVA, or one-way ANOVA followed by Bonferroni-Dunn test unless noted otherwise. Values in tables and graphs were expressed as mean SEM.

\section{Histological analysis}

Tissue preparation for measuring an axonal diameter and electron microscopy. Mice at 2 months of age were killed by transcardial perfusion with a mixed solution of $2 \%$ paraformaldehyde and $2.5 \%$ glutaraldehyde in $0.1 \mathrm{~m}$ phosphate-buffered solution, $\mathrm{pH}$ 7.4. The cervical spinal cords were taken out, followed by immersion with the same fixative overnight. The specimens were postfixed with $1 \%$ osmic acid in the same solution described above, followed by dehydration and embedding into epon in a standard manner.

Measurement of axonal diameter and myelin sheath dimension. We measured the axonal diameter of VRST ( $p l p 1^{\text {tg/- }}$ mice, $n=2$; Wt littermates, $n=2$ ). Transverse sections of C 3 spinal cord with $1 \mu \mathrm{m}$ thickness were stained with toluidine blue and photographed through an Olympus BX51 microscope with a $100 \times$ objective. Optimal brightness and gray scale pixel values were adjusted so as to provide the sharpest discrimination of the myelin/axon border. Axon and myelin profiles were traced in nonoverlapping contiguous fields. These traced images were converted to binary images. The area and short axis of myelinated axons were then measured using MATLAB (Media Cybernetics). Every third myelinated axon was sampled in five randomly chosen fields.

Electron microscopic analysis. We examined the paranodal structure of C3 ventral funiculus using a Philips EM208S electron microscope (Philips, Eindhoven).

Immunohistochemical analysis. Mice were perfused with $4 \%$ paraformaldehyde in $0.1 \mathrm{M}$ phosphate buffer, $\mathrm{pH} 7.4$ ( $p l p 1^{t g /-}$ mice, $n=3$; Wt littermates, $n=3$ ). Brains and spinal cords were collected, frozen, and cut in coronal and longitudinal sections, respectively. Immunostaining with contactin-associated protein (Caspr)- and Kv1.2-specific antibodies was conducted to examine paranodal structure as described previously (Ishibashi et al., 2002). The polyclonal rabbit antibody against Caspr (kind gift from Dr. Peles, The Weizmann Institute of Science, Rehovot,
Israel) and the mouse monoclonal antibody against Kv1.2 (Upstate Biotechnology) were used at dilutions of 1:2000 and 1:200, respectively. Alexa 488-conjugated goat anti-rabbit and Alexa 594-conjugated goat anti-mouse secondary antibodies (Molecular Probes) were used.

Images were captured with a laser-scanning microscope LSM510 or LSM5 PASCAL (Carl Zeiss). Digitized images $(73.1 \times 73.1 \mu \mathrm{m})$ were analyzed using MATLAB. To convert the intensity images to binary images, we used the method which chooses the threshold to minimize the intraclass variance of the thresholded black and white pixels (Otsu, 1979). Before measuring cluster lengths and area, we preserved only paired Caspr signals and omitted other invalid signals. We then calculated the length and area of Caspr cluster signal by MATLAB according to the method of Haralick (1992).

Statistical analysis. Statistical analysis of the quantitative histological results was conducted using software STATISTICA (STATISTICATM; StatSoft). Data did not generally fit a normal distribution (verified by Kolmogorov-Smirnoff test), therefore nonparametric statistical testing was performed including the Kruskal-Wallis test, Mann-Whitney U test, or Scheffé post hoc comparison as dictated by the comparison required. If data fit a normal distribution, we used Student's $t$ test.

\section{Results}

\section{Two-month-old $p l p 1^{\text {tg/- }}$ mice show abnormal behavior}

Previous reports described that 2-month-old $p l p 1^{t g /-}$ mice had neither symptoms nor histological signs of demyelination (Inoue et al., 1996; Ishibashi et al., 2003). However, CVs were significantly decreased in 2-month-old $p l p 1^{t g /-}$ mice (Tanaka et al., 2006). Therefore, this mouse provided us with a unique opportunity to analyze the behavioral abnormalities when CV was reduced by half. To do this, we performed a comprehensive behavioral analysis.

Our analysis examined physical characteristics, motor and sensory motor reflexes, nociception, depression-related behavior, anxiety-related behavior, and memory in the $p l p 1^{\text {tg/- }}$ transgenic mice. The results are summarized in Table 1 and Fig. 1. In the Porsolt forced swim test (test for depression related behavior), 16 of $21 p l p 1^{t g /-}$ mice exhibited tonic posture, and were drowning during the trials; one mouse also died after the trial. Therefore, immobility analysis was not conducted. No significant differences were observed in physical characteristics, motor function, open field tests, or pain nociception, between $p l p 1^{t g /-}$ and Wt mice. Thus, $p l p 1^{t g /-}$ mice are basically normal, which permit us to perform tasks to evaluate much higher brain functions at this age.

In the light/dark transition test, the number of transitions between the two boxes significantly decreased in $p l p 1^{\text {tg/- }}$ mice relative to $\mathrm{Wt}$ mice, whereas stay time in light or total distance traveled was not significantly different (Table 1). These results suggest altered anxiety-like behaviors in the $p l p 1^{t g /-}$ mice. We also performed the elevated plus maze test as another measurement for anxiety. The percentage of entries into the open arms of the maze, and the percentage of time spent on those arms were significantly greater in $p l p 1^{\text {tg/- }}$ mice compared with control $\mathrm{Wt}$ mice (Table 1). Although this result seems to indicate that the subjects show less fear, which is in contrast to the above results, these results are consistent with the idea that the $p l p 1^{t g /-}$ mice exhibit abnormal anxiety-like behaviors.

In the social interaction test, the number of total contacts tended to decrease in $p l p 1^{t g /-}$ mice but was not significant (Table 1).

The prepulse inhibition test is commonly used for measuring schizophrenic behavior (Braff and Geyer, 1990). The $p l p 1^{\text {tg/- }}$ mice showed a mildly impaired prepulse inhibition (Table 1).

We also used the Barnes spatial navigation task as a test for learning ability. This task requires the mouse to learn distal envi- 
Table 1. General physical characteristics, sensory/motor functions, depressionrelated behaviors, and anxiety-related behaviors of $p / p 1^{\text {tg/-}}$ mice and Wt mice

Wt

$p / p 1^{t g /-}$

\begin{tabular}{|c|c|c|}
\hline \multicolumn{3}{|l|}{ Physical characteristics } \\
\hline Weight (g) & $28.2( \pm 0.6)$ & $28.0( \pm 0.4)$ \\
\hline Rectal temperature $\left({ }^{\circ} \mathrm{C}\right)$ & $36.4( \pm 0.1)$ & $36.2( \pm 0.1)$ \\
\hline \multicolumn{3}{|l|}{ Motor test } \\
\hline Wire hang (latency to fall) (s) & $50.6( \pm 2.7)$ & $56.2( \pm 2.3)$ \\
\hline Grip strength (N) & $0.7( \pm 0.0)$ & $0.8( \pm 0.0)$ \\
\hline \multicolumn{3}{|l|}{ Open field test } \\
\hline Total distance for $30 \mathrm{~min}(\mathrm{~cm})$ & $3967.4( \pm 269.2)$ & $3432.7( \pm 282.7)$ \\
\hline Center stay time for $30 \mathrm{~min}(\mathrm{~s})$ & $7.3( \pm 0.8)$ & $8.0( \pm 0.7)$ \\
\hline Vertical activity for $30 \mathrm{~min}$ & $422.4( \pm 43.7)$ & $352.4( \pm 27.6)$ \\
\hline \multicolumn{3}{|c|}{ Anxiety-related behavior (Light/dark test) } \\
\hline Distance traveled in light $(\mathrm{cm})$ & $909.8( \pm 59.4)$ & $865.5( \pm 67.3)$ \\
\hline Distance traveled in dark (cm) & $1382.7( \pm 67.2)$ & $1238.0( \pm 41.0)$ \\
\hline Light/dark transitions & $26.8( \pm 1.9)$ & $21.6( \pm 1.5)^{*}$ \\
\hline Total time in light (s) & $247.2( \pm 13.5)$ & $257.4( \pm 14.8)$ \\
\hline \multicolumn{3}{|l|}{ Pain test } \\
\hline Hot plate (latency) (s) & $6.7( \pm 0.6)$ & $7.0( \pm 0.5)$ \\
\hline \multicolumn{3}{|l|}{ Social interaction in novel environment } \\
\hline Distance traveled $(\mathrm{cm})$ & $3131.2( \pm 130.7)$ & $2972.1( \pm 179.9)$ \\
\hline Number of total contacts & $60.9( \pm 2.9)$ & $55.1( \pm 4.5)$ \\
\hline Mean duration/contact (s) & $1.8( \pm 0.2)$ & $1.7( \pm 0.2)$ \\
\hline \multicolumn{3}{|l|}{ Sensory motor reflex } \\
\hline \multicolumn{3}{|c|}{ Pepulse inhibition (\%; startle stimulus $=110 \mathrm{~dB}$ ) } \\
\hline Prepulse intensity $=74 \mathrm{~dB}$ & $40.2( \pm 6.4)$ & $34.2( \pm 7.0)$ \\
\hline Prepulse intensity $=78 \mathrm{~dB}$ & $56.3( \pm 5.1)$ & $15.0( \pm 20.8)^{\#}$ \\
\hline \multicolumn{3}{|c|}{ Pepulse inhibition (\%; startle stimulus $=120 \mathrm{~dB})$} \\
\hline Prepulse intensity $=74 \mathrm{~dB}$ & $27.5( \pm 4.7)$ & $16.3( \pm 5.5)$ \\
\hline Prepulse intensity $=78 \mathrm{~dB}$ & $39.2( \pm 7.8)$ & $24.6( \pm 5.1)^{\#}$ \\
\hline \multicolumn{3}{|l|}{ Elevated plus-maze } \\
\hline Entries into open arms (\%) & $12.2( \pm 2.1)$ & $24.5( \pm 2.8)^{\#}$ \\
\hline Time spent on open arms (\%) & $4.7( \pm 1.2)$ & $10.9( \pm 1.7)^{\#}$ \\
\hline Total distance traveled $(\mathrm{cm})$ & $1439.6( \pm 79.2)$ & $1447.7( \pm 69.9)$ \\
\hline Total entry in arms & $25.3( \pm 1.9)$ & $28.6( \pm 1.9)$ \\
\hline
\end{tabular}

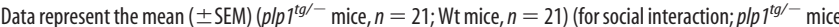
$n=10$; Wt mice, $n=9, n$ indicates number of pairs; for prepulse inhibition, $p l p 1^{\text {tg/ }}$ - mice, $n=20$; Wt mice, $n=$ 20). Statistical differences are ${ }^{*} p<0.05$ and ${ }^{\#} p<0.005$.

ronmental room cues to locate an escape hole. One of the $p l p 1^{t g /-}$ mice did not move so that this was omitted from the analysis. Acquisition trials revealed that the latency to reach the target above the escape box, the distance to reach the target, and errors to reach the target had no significant difference between $\mathrm{Wt}$ and $p l p 1^{t g /-}$ mice $\left(F_{(1,15)}=1.275, p=0.2766 ; F_{(1,15)}=0.001, p=\right.$ $\left.0.9771 ; F_{(1,15)}=0.925, p=0.3514\right)$. However, in the probe test, there was a significant difference. Wt mice spent significantly more time around the target than around any other holes when the probe test was conducted $1 \mathrm{~d}$ after the acquisition trials (Fig. 1A). $P$-values were all under $10^{-3}$ for the target versus any of the other holes, which were positioned at angles $\pm 30^{\circ}, \pm 60^{\circ}, \pm 90^{\circ}$, $\pm 120^{\circ}, \pm 150^{\circ}$ or $180^{\circ}$ from the target. The $p l p 1^{\text {tg } /-}$ mice did not spend much time on the target compared with the other holes. The number of errors to reach the target in the probe test was significantly greater in $p l p 1^{t g /-}$ mice (Wt mice, $0.22 \pm 0.15$; $p l p 1^{\text {tg } /-}$ mice, $\left.2.75 \pm 1.22 \mathrm{~cm} ; F_{(1,15)}=4.773, p=0.0452\right)$. The $p l p 1^{\text {tg/- }}$ mice spent a smaller percentage of time in the target than $\mathrm{Wt}$ mice $\left(35.2 \pm 7.6 \%\right.$ for $\mathrm{Wt} ; 17.0 \pm 3.1 \%$ for $p l p 1^{\text {tg }}$ - mice, $\left.F_{(1,15)}=4.445, p=0.0522\right) \cdot p l p 1^{t g /-}$ mice also showed a tendency to travel greater distances to reach the target (Wt mice, $46.4 \pm 3.1$ $\mathrm{cm} ; p l p 1^{\text {tg/- }}$ mice, $\left.102.3 \pm 31.0 \mathrm{~cm} ; F_{(1,15)}=3.619, p=0.0765\right)$, although these values were not statistically significant. These results indicate that selectivity of spatial search was severely impaired in $p l p 1^{\text {tg/- }}$ mice.
We next examined a T-maze forced alternation task, which is a task of working memory. During training sessions, $p l p 1^{\text {tg/- }}$ mice showed significantly less correct choices made than wildtype mice $\left(F_{(1,43)}=5.943, p=0.019\right.$, genotype effect; from first to 15 th session) (Fig. $1 B$ ). To increase the difficulty of the task, a delay period (10, 30, $60 \mathrm{~s})$ was applied. Under these conditions, there was significant difference in the percentage of correct choices made between $p l p 1^{\text {tg/- }}$ and wild-type mice (30 s delay, $F_{(1,43)}=6.534, p=0.014 ; 60$ s delay, $\left.F_{(1,43)}=5.563, p=0.023\right)$ (Fig. 1C). These results supported that working memory was impaired in $p l p 1^{t g /-}$ mice.

In conclusion, the $p l p 1^{t g /-}$ mice display cognitive impairment and abnormal anxiety-like behavior. Since the reduced $\mathrm{CV}$ in the $p l p 1^{t g /-}$ mice could cause these abnormal behaviors at 2 months of age, we studied mechanisms underlying this abnormal saltatory conduction.

\section{Axonal diameter and myelin thickness are altered in plp $^{\text {tg/- }}$ mice}

Five factors determine CVs: fiber diameter, myelin thickness, internodal distance, temperature, and nodal properties (Waxman, 1980). We first examined axonal diameter and myelin thickness in the VRST of two $p l p 1^{t g /-}$ mice and two Wt mice at 2 months of age. Figure 2, $A$ and $B$, shows toluidine blue staining of the VRST in Wt and $p l p 1^{t g /-}$ mice, respectively. We found two differences between wild-type and $p l p 1^{\text {tg/- }}$ mice: first, axonal diameters of $p l p 1^{t g /-}$ mice were relatively smaller than those of $\mathrm{Wt}$ mice $(3.39 \pm 0.09 \mu \mathrm{m}, 625$ fibers in Wt mice; $1.98 \pm 0.06 \mu \mathrm{m}, 602$ fibers in $p l p 1^{t g /-}$ mice; $p<10^{-4}$, Mann-Whitney $U$ test). The number of medium and large diameter axons $(>3 \mu \mathrm{m})$ (Fig. $2 C$, asterisk) decreased, whereas the number of small diameter axons increased (Fig. $2 C,<3 \mu \mathrm{m}, \#$ ). Second, the myelin in $p l p 1^{t g /-}$ mice was thinner than that in Wt mice; a significant difference was observed in the G-ratio (the diameter of axon/the diameter of the axon plus myelin) $\left(0.70 \pm 0.01,602\right.$ fibers in $p l p 1^{\text {tg/- }}$ mice; $0.57 \pm 0.01,625$ fibers in Wt mice; $p<10^{-4}$, Mann-Whitney $U$ test).

\section{Caspr cluster spreads along the axon of the VRST in plp $^{\text {tg/- }}$ mice}

Next, we performed immunostaining of the VRST with antiCaspr and anti-Kv1.2 antibodies to understand whether a paranodal abnormality was the cause for the affected CV. In Wt mice, Caspr clusters were separate from Kv1.2 signals (Fig. $3 A$ ). In contrast, Caspr clusters were scattered and overlapped with Kv1.2 signals in $p l p 1^{\text {tg } /-}$ mice (Fig. $3 C$ ). Interestingly Caspr clusters in $p l p 1^{t g /-}$ mice were more elongated along the axons in the VRST compared with those in Wt mice (Fig. $3 B, D$ ).

Figure $3 E$ shows a histogram of the length of Caspr clusters in the VRST at 2 months of age. The spread of Caspr clusters in the VRST of $p l p 1^{\text {tg/- }}$ mice was significantly longer than that seen in Wt mice. Caspr cluster area in $p l p 1^{\text {tg/- }}$ mice was also significantly larger than that in Wt mice (data not shown). These data indicate the abnormal distribution of Caspr protein, and strongly suggest that the paranodal structural abnormality is accountable for aberrant Caspr distribution in the $p l p 1^{t g /-}$ mice.

\section{Two-month-old $p l p 1^{\text {tg/- }}$ mice have an abnormal paranodal structure}

To examine paranodal structure, we performed electron microscopic analysis in the VRST of $p l p 1^{t g /-}$ mice. We observed everted paranodal myelin loops ( 34 of 46 nodes, Fig. $4 E$ ), myelin outfolding ( 3 of 46 nodes, Fig. $4 F$ ) and myelin infolding ( 2 of 46 nodes, 


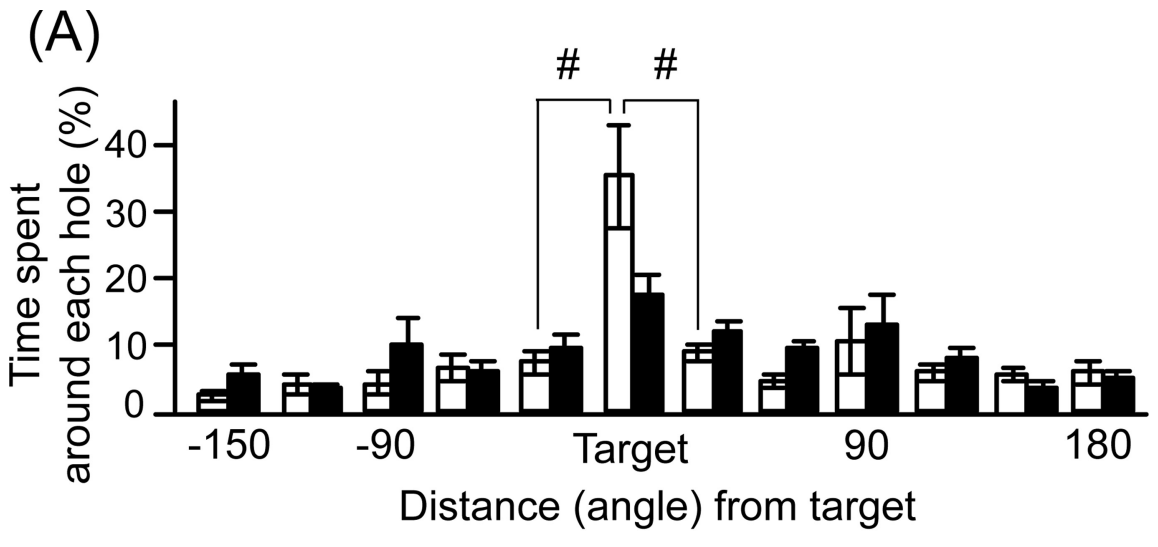

(B)

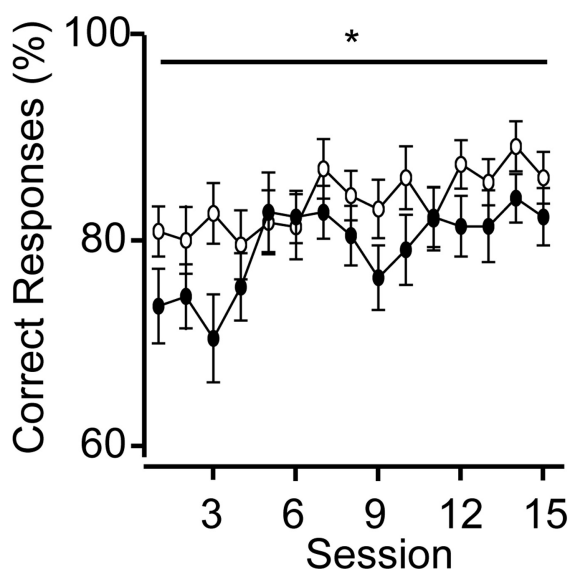

(C)

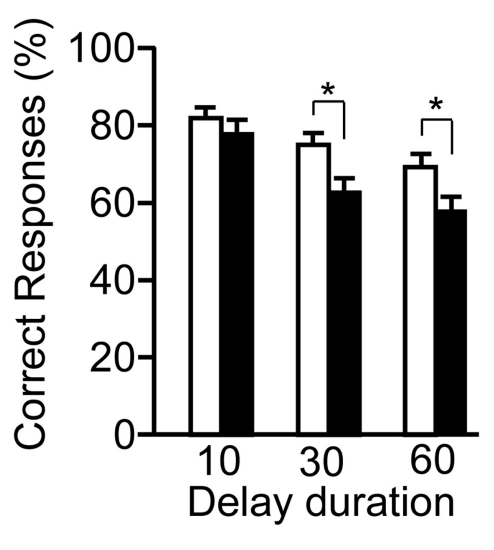

Figure 1. Impairment of cognitive task in the $p / p 1^{t g /-}$ mice. $A$ shows the results of probe test in Barnes spatial navigation task. Open bars indicate data of Wt mice, whereas black bars indicate those of $p / p 1^{t g /-}$ mice. Wt mice spent significantly more time around the target than around any other holes when the probe test was conducted $1 \mathrm{~d}$ after the acquisition trials $\left(\boldsymbol{A},{ }^{\#} p<0.01\right)$, whereas the $p / p 1^{t g /-}$ mice did not. $\boldsymbol{B}$ and $\boldsymbol{C}$ show the percentage of correct choices in T-maze forced alternation task. The $p / p 1^{t g /-}$ mice exhibited the lower correct responses during training session $\left(\boldsymbol{B},{ }^{*} p<0.05\right)$ and in 30 and 60 s delayed version $\left(\boldsymbol{C},{ }^{*} p<\right.$ 0.05), indicating that the working memory was impaired.

Fig. 4G). In contrast, we could not find any everted paranodal myelin loops, myelin infolding, or myelin outfolding in $\mathrm{Wt}$ mice (45 nodes examined, Fig. $4 A$ ). In addition, some of the paranodal loops from $p l p 1^{t g /-}$ mice (6 of 46 nodes) showed no septate-like junctions (transverse bands) between the axolemma and the apposing paranodal loops (Fig. 4C,D). We never observed the loss of transverse bands in Wt mice (Fig. $4 B$ ). The loss of the transverse bands is an abnormal phenotype; however, many paranodes still retained transverse bands in the $p l p 1^{t g /}$ mice, indicating that this defect is much milder than that seen in mutants lacking paranodal proteins (Poliak and Peles, 2003). Since this mouse will eventually lose paranodal junctions at later stages, it is highly likely that we are observing initial paranodal openings that lead to demyelination. We and others have observed that after a complete opening of the paranodal structure, the Caspr signal is spread evenly on axons (Ishibashi et al., 2002). Therefore, increased spread of Caspr localization, and an overlap of Caspr signal with that of potassium channels should indicate the initial delocalization of these proteins.

\section{Caspr clusters are disrupted in the corpus callosum of} $p l p 1^{\text {tg/- }}$ mice

We next performed immunostaining of the corpus callosum with anti-Caspr and anti-Kv1.2 antibodies to examine whether a para- nodal abnormality also existed in the forebrain of $p l p 1^{\text {tg/- }}$ mice. This brain region is intimately linked to schizophrenia. In Wt mice, intense Caspr signals segregated from Kv1.2 signals, similar to what we observed in the spinal cord. In contrast, intense Caspr signals were disrupted in $p l p 1^{t g /-}$ mice and only weak signals were diffusely located on the axons. Figure $5 C$ shows histograms of the length of intense Caspr signals in Wt and $p l p 1^{\text {tg/- }}$ mice. This result showed that Caspr length of $p l p 1^{\text {tg/- }}$ mice was significantly shorter than that of Wt mice in the corpus callosum $(1.07 \pm$ $0.50 \mu \mathrm{m}, 300$ clusters in $p l p 1^{\text {tg } /-}$ mice; $2.29 \pm 0.41 \mu \mathrm{m}, 300$ clusters in Wt mice; $p<0.01$, Student's $t$ test), when we focus on the intense signal. Therefore, a paranodal abnormality also exists in the forebrain. Shortening of the Caspr signal may indicate that the delocalization of Caspr started much earlier than in the spinal cord and the spread Caspr signal became undetectable by the immunostaining method used in this study.

\section{Discussion}

White matter abnormality and schizophrenia

Schizophrenia, which is characterized by hallucinations, delusions, disorganized thoughts, and various cognitive impairments, is one of the most devastating psychiatric disorders. Abnormal neurodevelopment, hyperdopaminergic, and hypoglutaminergic hypotheses have been offered to explain the etiology of the disease, but none by itself can explain the entire spectrum of its pathogenesis (Ross et al., 2006). Further, growing evidence from histological, genetic, and gene expression studies suggests that myelin and oligodendrocyte dysfunction contributes to the development of schizophrenia (Davis et al., 2003). mRNA levels of myelin proteolipid protein 1 ( $p l p 1$ ), a gene expressed in myelin, were found to be decreased in postmortem patient brains (Tkachev et al., 2003). Also, a weak association between plp1 and schizophrenia was also reported (Qin et al., 2005). Proteolipid protein 1 is the major protein constituent of CNS myelin, and is remarkably well conserved at the amino acid level in many mammalian species, including the mouse, rat, cow and human. None of the mutations found so far in $p l p 1$ can be tolerated by animals, and these mostly result in a severe dysmyelinating disease called Pelizaeus-Merzbacher disease in humans (MIM\# 312080). Only a minor population of mutations causes a much milder phenotype (spastic paraplegia type 2; MIM\# 300401). Duplication of the plp1 gene causes Pelizaeus-Merzbacher disease, whereas deletion of the plp1 gene shows very little effect on myelination. However, animals with no plp1 gene show axonal abnormalities (Garbern et al., 2002). Therefore, $p l p 1$ gene structure as well as its expression level has to be strictly regulated.

The diagnostic criteria for schizophrenia are defined in the DSM-IV-TR (American Psychiatric Association, 2000). Although many symptoms of the disorder are difficult to measure 
(A)

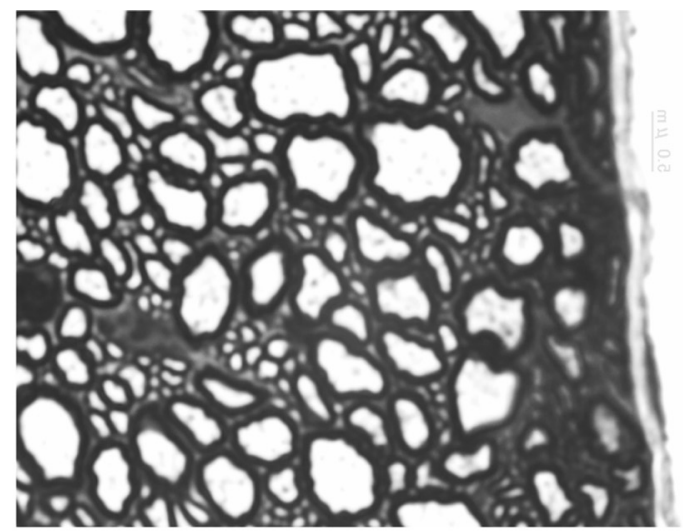

(B)

(C)
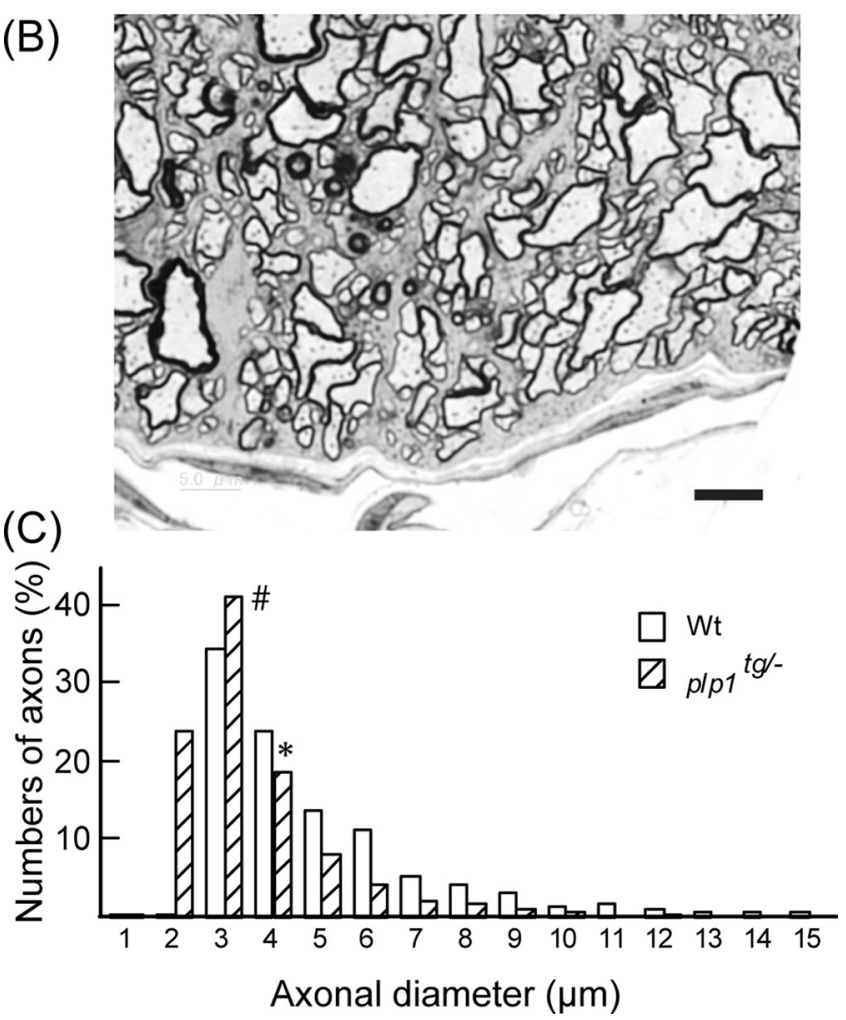

Figure 2. Axonal diameter changes in $p / p 7^{t g /-}$ mice. $A, B$, Transverse sections of $C 3$ spinal cord stained with toluidine blue to reveal the ventral funiculus in Wt mice $(\boldsymbol{A})$ and $p l p 1^{t g /-}$ mice $(\boldsymbol{B})$. Comparing $\boldsymbol{A}$ and $\boldsymbol{B}$, we found that axonal diameters in $p / p 1^{t g /-}$ mice were relatively smaller than those of Wt mice, and that the myelin in $p / p 1^{t g /-}$ mice was thinner than that in Wt mice. Scale bar: $10 \mu \mathrm{m}$. C, Histogram of myelinated axonal diameters showed that medium and large diameter axons ( $>3 \mu \mathrm{m})$ seemed to be replaced by small diameter fibers $(<3 \mu \mathrm{m})$ in $p / p 1^{t g /-}$ mice. The asterisk indicates the decreased number of axons with medium diameter, and the sharp indicates the increased number of axons with small diameter in $p l p 1^{t g /-}$ mice.

in rodents, some measurable behavioral abnormalities in schizophrenia, such as disruption in prepulse inhibition of startle response (PPI) and cognitive deficits, can be also assessed by the tests for rodents that mimic the ones for humans. Here we demonstrated that altered gene expression of the $p l p 1$ gene can result in abnormal behaviors (Table 1; Fig. 1). We observed PPI deficit, decreased working memory, and decreased spatial memory in the $p l p 1^{t g /-}$ mice in addition to altered anxiety-like behavior. PPI provides a measure of sensorimotor gating that can model the preattentive processing deficits that are observed in schizophrenic patients. PPI deficits can be directly measured in both humans and rodents, in essentially the same manner, and that measures of PPI are among the most frequently used paradigms
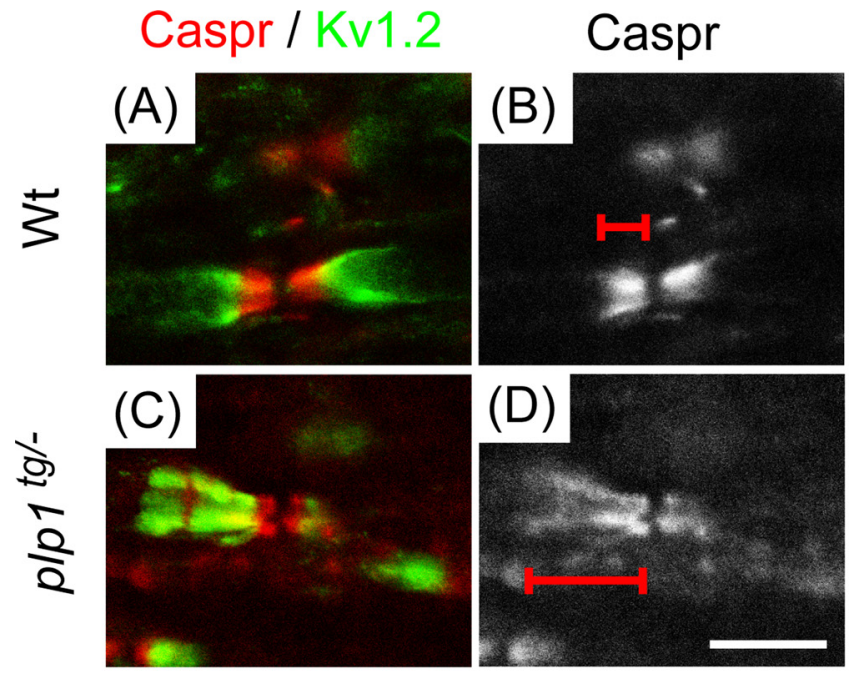

(E)

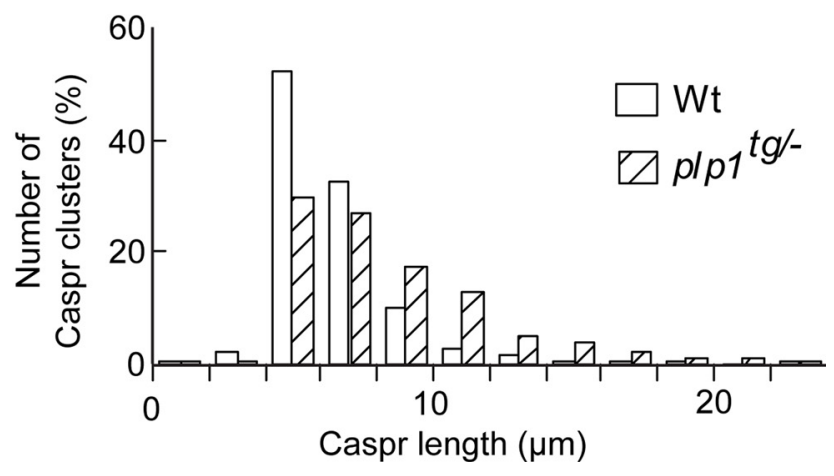

Figure 3. $\boldsymbol{A}-\boldsymbol{D}$, Localization of Caspr (red) and Kv1.2 (green) at the paranodal junctions and juxtaparanodal area, respectively, in 2-month-old Wt $(\boldsymbol{A})$ and $p / p 1^{t g /-}$ mice (C). Caspr clusters (white) at the paranodal junctions in $\mathrm{Wt}(\boldsymbol{B})$ and $p / p 7^{t g /-}$ mice $(\boldsymbol{D})$. Note the dispersed distribution of Caspr clusters in $p / p 7^{t g /-}$ mice (compare the red horizontal bars in $\boldsymbol{B}$ and $\boldsymbol{D}$ ). The signals of Caspr and Kv1.2 showed considerable overlap in the paranodal area of $p / p 1^{\text {tg/ }}$ - mice (C). Scale bar: $10 \mu \mathrm{m}$. $\boldsymbol{E}$, Histogram showing the distribution of the length of Caspr clusters in the VRST. The length of Caspr signals was elongated in $p / p 1^{t g /-}$ mice.

in animal models of schizophrenia (Braff and Geyer, 1990; Powell and Miyakawa, 2006).

Each phenotype is not typical to schizophrenia, but we thought that coincidence of three cognitive symptoms (decreased working memory, decreased sensorimotor gating (PPI deficit), and decreased spatial memory) was relevant to schizophrenia (Powell and Miyakawa, 2006). These cognitive symptoms should be observed in neurodevelopmental disorders, such as mental retardation, or neurodegenerative disorders, such as Alzheimer disease. However, the onset of plp1 expression is in the perinatal period, suggesting that neuronal migration and wiring events (that could be impaired in neurodevelopmental disorders) are normal in the $p l p 1^{t g /-}$ mice. In addition, the $p l p 1^{t g /-}$ mice never show the neuronal cell death. We think that the cognitive symptoms are due to the decreased CVs but not due to the wiring mismatch or the neuronal cell death.

The $p l p 1^{t g /-}$ mice did not show psychomotor agitation (relevant to positive symptom) and social withdrawal (relevant to negative symptom). In general, it is difficult to assess behaviors typical of a human disorder that is characterized by delusion and hallucination in a rodent model, since we do not have appropriate tests to address these typical behaviors. We think that other 

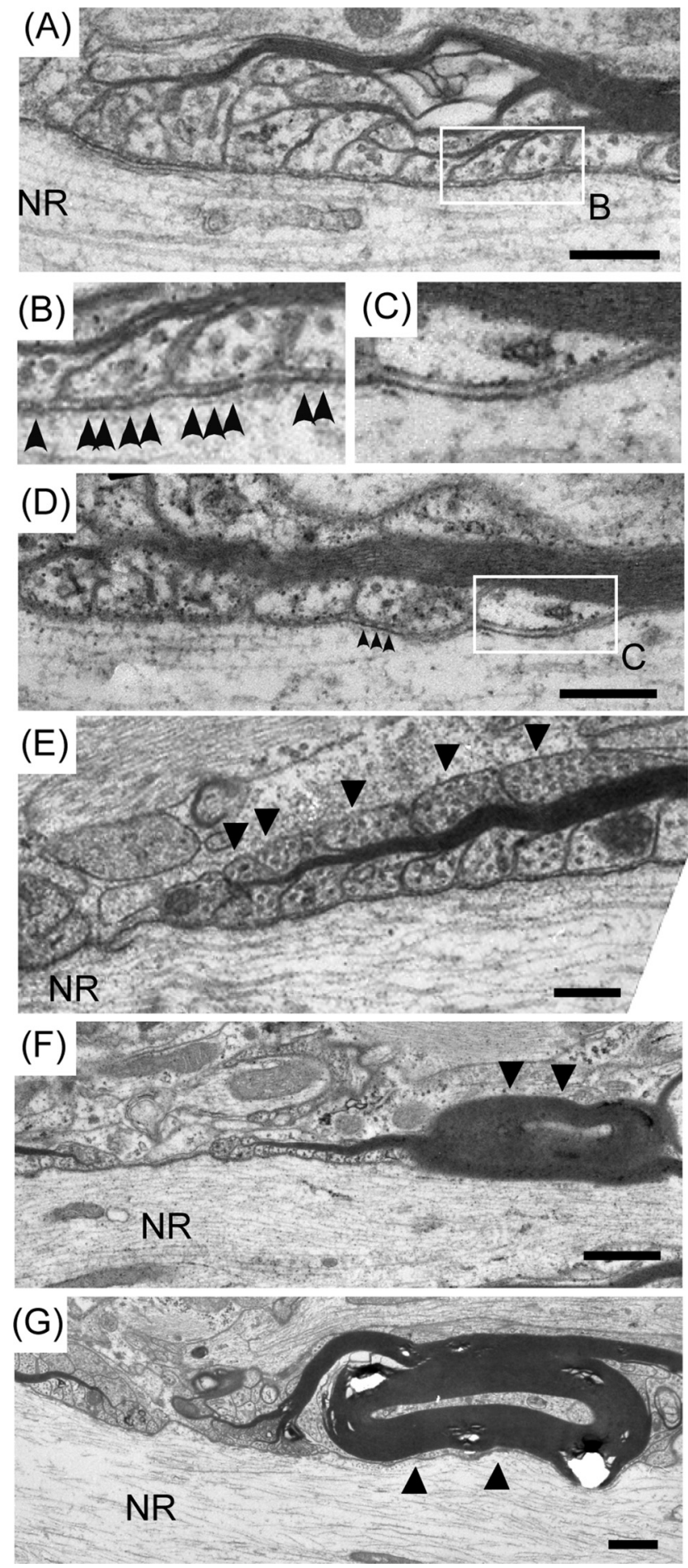

Figure 4. $\quad \boldsymbol{A}-\boldsymbol{D}$, Ultrastructure of paranode. $\boldsymbol{A}, \boldsymbol{D}$, Paranodal region of Wt $(\boldsymbol{A})$ and $p / p 7^{\mathrm{tg} /-}(\boldsymbol{D})$ mice. $\boldsymbol{B}, \boldsymbol{C}$, Magnified images of the white boxes in $\boldsymbol{A}$ and $\boldsymbol{C}$. Arrowheads in $\boldsymbol{B}$ show the septatelike junctions between the paranodal loops and the axolemma in a Wt mouse that has well formed and well preserved septate-like junctions (transverse bands). Arrowheads in $\boldsymbol{D}$ show the septate-like junctions in a $p / p 7^{\text {tg/- }}$ mouse; however, a loss of septate-like junctions was observed in $p / p 1^{\text {tg/- }}$ mice (C). $\boldsymbol{E}-\boldsymbol{G}$, Abnormal structures observed in $p / p 1^{\text {tg/- }}$ mice: arrowheads in $\boldsymbol{E}$ show an everted paranodal myelin loop; arrowheads in $\boldsymbol{F}$ show a myelin outfolding; arrowheads in $\boldsymbol{G}$ show a myelin infolding. NR: node of Ranvier. Scale bars: $(\boldsymbol{A}, \boldsymbol{D}), 300 \mathrm{~nm} ;(\boldsymbol{E}), 100 \mathrm{~nm} ;(\boldsymbol{F}, \boldsymbol{G}), 1 \mu \mathrm{m}$.

schizophrenia like behaviors could be underestimated by the lack of appropriate tests, or the $p l p 1^{\text {tg/- }}$ mice did not show positive and negative symptoms just as patients with schizophrenia do not manifest every possible symptom of the disease.
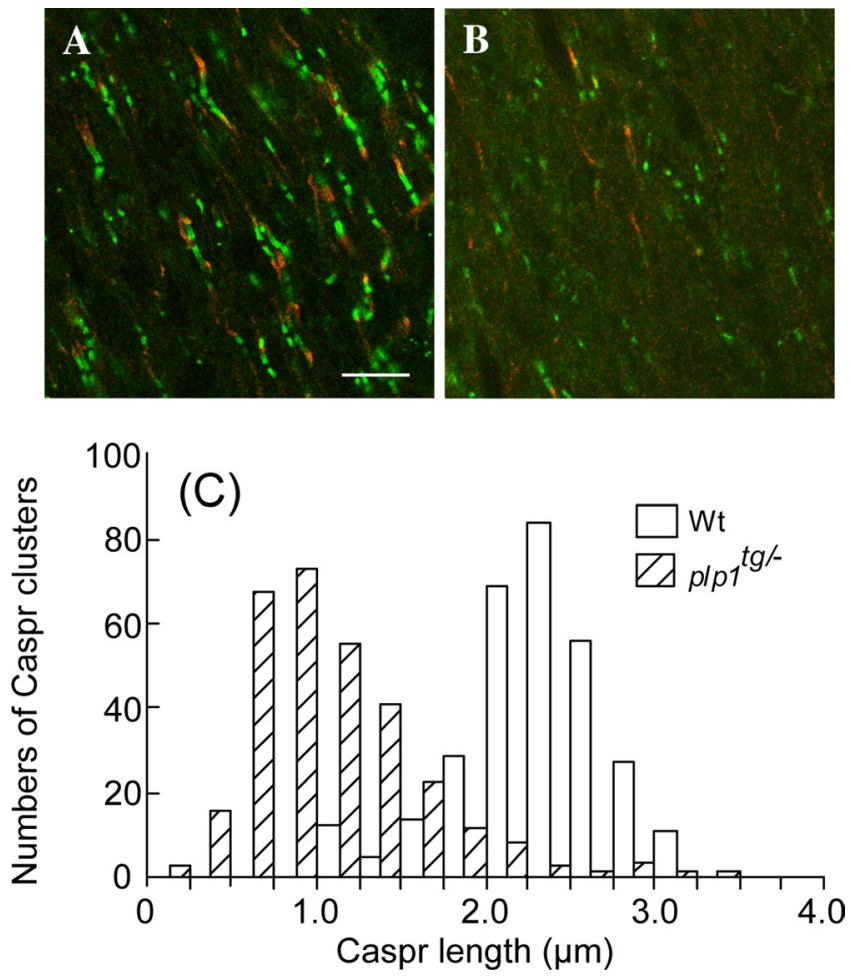

Figure 5. Abnormal Caspr and Kv1.2 clustering in the corpus callosum. $\boldsymbol{A}, \boldsymbol{B}$, Immunostaining for Caspr (green) and Kv1.2 (red) shows that $p / p 7^{\text {tg/- }}$ mice have many disrupted paranodes $(\boldsymbol{B})$ compared with those of Wt mice $(\boldsymbol{A})$. Note that intense Caspr signals were shortened and weak Caspr signals were scattered. Scale bar: $5 \mu \mathrm{m}$. C, Histogram showing the distribution of the length of intense Caspr signals in the corpus callosum ( $n=300$ each). Intense Caspr signals in $p l p 7^{t g /-}$ mice were shorter than those in Wt mice.

In terms of anxiety-like behavior, the $p l p 1^{\text {tg/- }}$ mice showed the opposite behavior: decrease in the number of transition in the light/dark transition test (suggesting increased anxiety) and increase in time spent in open arm in the elevated plus maze test (suggesting decreased anxiety). This discrepancy was difficult to be interpreted, but at least, the $p l p 1^{\text {tg/- }}$ mice exhibited abnormal responses in the stressful situation. Anxiety is not an important phenotype in diagnostic process and the affective disorder should be excluded when we diagnose the patient as schizophrenia according to DSM-IV criteria. However, the comorbidity of anxiety is frequently observed in the schizophrenic patients, so that we think the altered anxiety-like behavior is compatible with schizophrenia-like behavior in mice. Taken together, we concluded that our transgenic mice before demyelinating stage exhibited schizophrenia-related abnormal behavior.

Plp1 gene expression is mainly restricted to oligodendrocytes, indicating that a primary abnormality in white matter can lead to cognitive deficits. White matter changes, including an increased cell density in deep white matter (Akbarian et al., 1996; Kirkpatrick et al., 1999) and dysregulation of myelin-related gene expression (Hakak et al., 2001; Tkachev et al., 2003), have also been found in schizophrenic patients. Moreover, the late-onset form of metachromatic leukodystrophy often presents with symptoms of acute schizophrenia at early stages before the symptoms derived from demyelination appear (Davis et al., 2003). White matter changes may not be the sole cause for the development of schizophrenic symptoms, but more and more evidence is accumulating to indicate that this plays an important role in at least some subset of schizophrenia. However, it is still unknown 
how these white matter abnormalities are related to schizophrenia-like behaviors.

Here we propose a possible mechanism that will at least enable us to explain abnormal behavior in our mouse. (Our mouse model harbors an inserted transgene, thus it is possible that all these behavioral abnormalities are caused by the disruption of a gene, but because of the significance of white matter in schizophrenia as discussed above, we believe that this is less likely to have occurred.) Altered expression of myelin gene(s) causes a subtle abnormality in oligodendrocytes resulting in abnormal neuron-glia interaction at the paranode. This results in an altered axonal diameter, and a profound decrease in the CV. The extent of the decrease in $\mathrm{CV}$ would be distinct among fibers, and would lead to a failure in coordinated stimulation of a single neuron, which is necessary to integrate information from different neural circuits. This mechanism may be applicable to some of the schizophrenic patients as will be discussed below.

\section{Altered myelin gene expression as the cause for paranodal abnormality, smaller axonal diameter, and reduced conduction velocity}

Oligodendrocytes regulate regional expansion of axon caliber during development, independent of myelin formation (Sánchez et al., 1996). This glial signal to axons may be mediated by direct contact through paranodal junctions, or through humoral factors secreted by oligodendrocytes (Kaplan et al., 1997). Another study examined the axonal diameter, myelin thickness, and $g$ ratio of peripheral nerves in transgenic mice overexpressing peripheral myelin protein 22 (PMP22) mRNA by $210 \%$ (RobagliaSchlupp et al., 2002). The authors showed that these mice had smaller fiber diameters and thinner myelin compared with those of Wt mice at P16, and that larger diameter axons seemed to remain unmyelinated. Thus, defects in glial cells can result in abnormal neuron-glia interactions, and cause changes in the axonal diameter, myelin thickness, and g ratio. Abnormal paranodal junctions found in the 2-month-old $p l p 1^{\text {tg/- }}$ mice (Figs. 3 and 4) indicate abnormal neuron-glia interactions, which result in axonal abnormalities. These changes can cause reduction in the $\mathrm{CV}$, which is determined by five factors: fiber diameter, myelin thickness, internode distance, temperature, and nodal properties (Waxman, 1980).

\section{Reduced conduction velocity and abnormal behavior}

We have no direct evidence showing that the abnormal saltatory conduction caused the abnormal behaviors observed in our study. However, the electrophysiological abnormality can explain many symptoms found in schizophrenic patients. Many learning processes are "associative", which require input from two neuronal circuits to reach a single neuron simultaneously. When the CVs of various axons are reduced to a different degree, simultaneous input cannot be achieved. This can cause disorganized thought and various cognitive impairments.

In conclusion, in one of the mouse lines with myelin/oligodendrocyte defects ( $p l p 1^{t g /-}$ mice), we found abnormal neuronglia interactions and electrophysiological abnormalities in the CNS. We also observed abnormal behavior related to schizophrenia in this mouse. Our present and previous results strongly suggest that defects in white matter can result in decreased CV in human schizophrenic patients, and that this decrease in CV may be one of the causes of schizophrenia. It will be most informative to measure CV in the CNS (especially in the prefrontal cortex) of schizophrenic patients with myelin/oligodendrocyte defects, and to correlate it with their symptoms.

\section{References}

Akbarian S, Kim JJ, Potkin SG, Hetrick WP, Bunney WE Jr, Jones EG (1996) Maldistribution of interstitial neurons in prefrontal white matter of the brains of schizophrenic patients. Arch Gen Psychiatry 53:425-436.

American Psychiatric Association (2000) Diagnostic and statistical manual of mental disorders: DSM-IV-TR, 4th ed. Washington DC: American Psychiatric Association.

Braff DL, Geyer MA (1990) Sensorimotor gating and schizophrenia. Human and animal model studies. Arch Gen Psychiatry 47:181-188.

Crawley JN (2000) What's wrong with my mouse? Behavioral phenotyping of transgenic and knockout mice. New York: Wiley.

Davis KL, Stewart DG, Friedman JI, Buchsbaum M, Harvey PD, Hof PR, Buxbaum J, Haroutunian V (2003) White matter changes in schizophrenia: evidence for myelin-related dysfunction. Arch Gen Psychiatry 60:443-456.

Garbern JY, Yool DA, Moore GJ, Wilds IB, Faulk MW, Klugmann M, Nave KA, Sistermans EA, van der Knaap MS, Bird TD, Shy ME, Kamholz JA, Griffiths IR (2002) Patients lacking the major CNS myelin protein, proteolipid protein 1, develop length-dependent axonal degeneration in the absence of demyelination and inflammation. Brain 125:551-561.

Hakak Y, Walker JR, Li C, Wong WH, Davis KL, Buxbaum JD, Haroutunian V, Fienberg AA (2001) Genome-wide expression analysis reveals dysregulation of myelination-related genes in chronic schizophrenia. Proc Natl Acad Sci U S A 98:4746-4751.

Haralick S (1992) Computer and robot vision, Vol I. Reading, MA: Addison-Wesley.

Inoue Y, Kagawa T, Matsumura Y, Ikenaka K, Mikoshiba K (1996) Cell death of oligodendrocytes or demyelination induced by overexpression of proteolipid protein depending on expressed gene dosage. Neurosci Res 25:161-172.

Ishibashi T, Dupree JL, Ikenaka K, Hirahara Y, Honke K, Peles E, Popko B, Suzuki K, Nishino H, Baba H (2002) A myelin galactolipid, sulfatide, is essential for maintenance of ion channels on myelinated axon but not essential for initial cluster formation. J Neurosci 22:6507-6514.

Ishibashi T, Ikenaka K, Shimizu T, Kagawa T, Baba H (2003) Initiation of sodium channel clustering at the node of Ranvier in the mouse optic nerve. Neurochem Res 28:117-125.

Kagawa T, Ikenaka K, Inoue Y, Kuriyama S, Tsujii T, Nakao J, Nakajima K, Aruga J, Okano H, Mikoshiba K (1994) Glial cell degeneration and hypomyelination caused by overexpression of myelin proteolipid protein gene. Neuron 13:427-442.

Kaplan MR, Meyer-Franke A, Lambert S, Bennett V, Duncan ID, Levinson SR, Barres BA (1997) Induction of sodium channel clustering by oligodendrocytes. Nature 386:724-728.

Kirkpatrick B, Conley RC, Kakoyannis A, Reep RL, Roberts RC (1999) Interstitial cells of the white matter in the inferior parietal cortex in schizophrenia: an unbiased cell-counting study. Synapse 34:95-102.

Otsu N (1979) A threshold selection method from gray-level histogram. IEEE Trans Syst 9:62-66.

Poliak S, Peles E (2003) The local differentiation of myelinated axons at nodes of Ranvier. Nat Rev Neurosci 4:968-980.

Powell CM, Miyakawa T (2006) Schizophrenia-relevant behavioral testing in rodent models: a uniquely human disorder? Biol Psychiatry 59:1198-1207.

Qin W, Gao J, Xing Q, Yang J, Qian X, Li X, Guo Z, Chen H, Wang L, Huang X, Gu N, Feng G, He L (2005) A family-based association study of PLP1 and schizophrenia. Neurosci Lett 375:207-210.

Rasband MN, Kagawa T, Park EW, Ikenaka K, Trimmer JS (2003) Dysregulation of axonal sodium channel isoforms after adult-onset chronic demyelination. J Neurosci Res 73:465-470.

Robaglia-Schlupp A, Pizant J, Norreel JC, Passage E, Sabéran-Djoneidi D, Ansaldi JL, Vinay L, Figarella-Branger D, Lévy N, Clarac F, Cau P, Pellissier JF, Fontés M (2002) PMP22 overexpression causes dysmyelination in mice. Brain 125:2213-2221.

Ross CA, Margolis RL, Reading SA, Pletnikov M, Coyle JT (2006) Neurobiology of schizophrenia. Neuron 52:139-153.

Sánchez I, Hassinger L, Paskevich PA, Shine HD, Nixon RA (1996) Oligodendroglia regulate the regional expansion of axon caliber and local accumulation of neurofilaments during development independently of myelin formation. J Neurosci 16:5095-5105.

Takao K, Toyama K, Nakanishi K, Hattori S, Takamura H, Takeda M, Miyakawa T, Hashimoto R (2008) Impaired long-term memory retention 
and working memory in sdy mutant mice with a deletion in Dtnbp1, a susceptibility gene for schizophrenia. Mol Brain 1:11.

Tanaka H, Ikenaka K, Isa T (2006) Electrophysiological abnormalities precede apparent histological demyelination in the central nervous system of mice overexpressing proteolipid protein. J Neurosci Res 84:1206-1216.

Tkachev D, Mimmack ML, Ryan MM, Wayland M, Freeman T, Jones PB, Starkey M, Webster MJ, Yolken RH, Bahn S (2003) Oligodendrocyte dysfunction in schizophrenia and bipolar disorder. Lancet 362:798-805.

Waxman SG (1980) Determinants of conduction velocity in myelinated nerve fibers. Muscle Nerve 3:141-150.

Waxman SG, Bangalore L (2005) Myelin function and salutatory conduc- tion. In: Neuroglia, Ed 2 (Kettenmann H, Ransom BR, eds), pp 273-284. New York: Oxford UP.

Yamasaki N, Maekawa M, Kobayashi K, Kajii Y, Maeda J, Soma M, Takao K, Tanda K, Ohira K, Toyama K, Kanzaki K, Fukunaga K, Sudo Y, Ichinose H, Ikeda M, Iwata N, Ozaki N, Suzuki H, Higuchi M, Suhara T, et al. (2008) Alpha-CaMKII deficiency causes immature dentate gyrus, a novel candidate endophenotype of psychiatric disorders. Mol Brain 1:6.

Yamazaki Y, Hozumi Y, Kaneko K, Sugihara T, Fujii S, Goto K, Kato H (2007) Modulatory effects of oligodendrocytes on the conduction velocity of action potentials along axons in the alveus of the rat hippocampal CA1 region. Neuron Glia Biol 3:325-334. 